Article

\title{
The Slippery Path to Total Presence: How Omnidirectional Virtual Reality Treadmills Influence the Gaming Experience
}

\author{
Lars-Ole Wehden *, Felix Reer, Robin Janzik, Wai Yen Tang and Thorsten Quandt \\ Department of Communication, University of Muenster, 48143 Muenster, Germany; \\ E-Mails: lars-ole.wehden@uni-muenster.de (L.-O.W.), felix.reer@uni-muenster.de (F.R.), \\ robin.janzik@uni-muenster.de (R.J.), tangw@uni-muenster.de (W.Y.T.), thorsten.quandt@uni-muenster.de (T.Q.) \\ * Corresponding author
}

Submitted: 17 April 2020 | Accepted: 15 June 2020 | Published: 6 January 2021

\begin{abstract}
Researchers, game designers, and consumers place great hopes into the potential benefits of virtual reality (VR) technology on the user experience in digital games. Indeed, initial empirical research has shown that VR technology can improve the gaming experience in a number of ways compared to traditional desktop gaming, for instance by amplifying immersion and flow. However, on the downside, a mismatch between physical locomotion and the movements of the avatar in the virtual world can also lead to unpleasant feelings when using VR technology-often referred to as cybersickness. One solution to this problem may be the implementation of novel passive repositioning systems (also called omnidirectional treadmills) that are designed to allow a continuous, more natural form of locomotion in VR. In the current study, we investigate how VR technology and the use of an omnidirectional treadmill influence the gaming experience. Traditional desktop gaming, VR gaming, and omnidirectional treadmill gaming are compared in a one-factorial experimental design $(N=203)$. As expected, we found that VR gaming on the one hand leads to higher levels of flow, presence, and enjoyment, but at the same time also is accompanied by higher levels of cybersickness than traditional desktop gaming. The use of the omnidirectional treadmill did not significantly improve the gaming experience and also did not reduce cybersickness. However, this more physically demanding form of locomotion may make omnidirectional treadmills interesting for exergame designers.
\end{abstract}

\section{Keywords}

cybersickness; digital games; experimental research; gaming experience; locomotion; omnidirectional treadmill; passive repositioning systems; virtual reality

\section{Issue}

This article is part of the issue "Games and Communication-Quo Vadis?" edited by Marko Siitonen (University of Jyväskylä, Finland), Felix Reer (University of Muenster, Germany) and Teresa de la Hera (Erasmus University Rotterdam, The Netherlands).

(C) 2021 by the authors; licensee Cogitatio (Lisbon, Portugal). This article is licensed under a Creative Commons Attribution 4.0 International License (CC BY).

\section{Introduction}

The market introduction of affordable virtual reality (VR) devices like the Oculus Rift or the HTC VIVE is currently a much-discussed development in the field of gaming. Market researchers expect the revenue of VR products to more than quadruple between 2018 and 2022 (Superdata, 2019). Gaming plays an important role within the market for immersive technology, making up for a substantial part of its income, accounting for $43 \%$ in 2018 (Viar360, 2019).

While the industry is expecting a positive effect on hardware and software sales, players of VR games hope for a more thrilling and realistic gaming experience. Empirical research has indeed shown that VR technology can significantly improve the gaming experience in various ways and, for example, lead to increases in game enjoyment (Klimmt, Possler, \& Steger, 2018; 
Shelstad, Smith, \& Chaparro, 2017), flow (Tan, Leong, Shen, Dubravs, \& Si, 2015), or immersion (Martel et al., 2015). However, there are also specific issues related to current VR systems, like the frequently reported cybersickness problem (Dennison, Wisti, \& D'Zmura, 2016; McCauley \& Sharkey, 1992; Yildirim, 2019).

In recent years, novel ways of locomotion in VRamong them passive repositioning systems in the form of omnidirectional treadmills - have been developed (Hale \& Stanney, 2014). They were designed to allow for a more natural way of movement within VR by overcoming the problematic discrepancy between the available physical tracking space and the potentially unlimited virtual environment. These systems are expected to reduce cybersickness that results from an illusory mismatch between actual and perceived movement (Shafer, Carbonara, \& Korpi, 2019). However, empirical research on the effects of using omnidirectional VR treadmills is still scarce.

The present study follows two main goals: First, we aim to confirm findings from previous studies that indicated a positive impact of VR technology on the gaming experience. And second, we follow an exploratory approach by investigating the effects of using a current commercial omnidirectional locomotion system (the Virtuix Omni). Particularly, we are interested in whether using the treadmill further improves the gaming experience and whether it may solve the cybersickness problem.

\section{VR and the Gaming Experience}

Several empirical studies assessed the impact of VR on players' gaming experiences. Some of these studies primarily focused on technical aspects, such as the visual quality and the performance of VR controllers. For example, Shelstad et al. (2017) showed in an experiment that players of a VR game reported a higher perceived sound and graphics quality than participants that played the same game on a desktop computer. In contrast, user performance experiments showed that players performed significantly worse in control and accuracy tasks (e.g., jumping, aiming, wall-hits) in VR compared to playing in a desktop setting (Martel et al., 2015; Tan et al., 2015).

Of greater interest for the current study is research that examined more complex, psychological constructs. Two often studied variables in this context are presence and immersion (Witmer \& Singer, 1998). Presencewhen used in a media context also called telepresenceis defined as "the extent to which one feels present in the mediated environment, rather than in the immediate physical environment" (Steuer, 1992, p. 76). Likewise, immersion in the gaming context describes the:

Sense of being 'in a game' where a person's thoughts, attention and goals are all focused around the game as opposed to attending to being concerned with anything else, such as what is going on in the room around them. (Sanders \& Cairns, 2010, p. 160)
Empirical studies have shown that gaming in VR positively affects reported levels of presence and immersion. Klimmt et al. (2018) showed in an experiment that VR gaming amplified presence as compared to traditional desktop gaming. Martel et al. (2015) and Tan et al. (2015) found that the level of reported immersion was significantly higher when a game was played in VR as compared to a desktop computer setting.

A related concept is flow. As a measure for general enjoyment and intrinsic motivation that can occur in relation to many activities (e.g., work, sports), flow is described as a state of total pleasure and optimal experience (Csíkszentmihályi, 1990). According to Sweetser and Wyeth (2005), a perfect match between skills and challenge is a major prerequisite of flow experiences. This makes flow an important concept to study in the context of digital games, as games provide players with an increasing level of difficulty, growing along with the skillset of the gamer. Flow has also been studied in relation to gaming in a VR context. Tan et al. (2015) showed that VR gaming induced higher levels of flow than desktop gaming. Accordingly, Shelstad et al. (2017) found that playing in VR led to higher levels of players' engrossment with the game, meaning that the game was able to hold players' attention and interest in a higher degree than gaming on a desktop computer.

Furthermore, experimental studies show that players of VR games reported higher enjoyment and overall satisfaction with the game when compared to players of desktop games (Klimmt et al., 2018; Shelstad et al., 2017). Even though novelty effects cannot be ruled out completely, these results further strengthen the assumption that gaming in VR could contribute to the formation of the above-mentioned optimal gaming experiences.

However, an important problem of VR gaming is cybersickness. Cybersickness is a form of motion sickness resulting from a mismatch between the illusory self-motion in the virtual environment and the actual external sensory information on the non-movement of the own body in physical space (McCauley \& Sharkey, 1992; Nilsson, Serafin, Steinicke, \& Nordahl, 2018). Cybersickness in VR is often caused by a lack of physical tracking space that prevents the user from unconstrained actual movement in a spatially vast virtual world. Therefore, movements have to be performed by pressing buttons or moving joysticks instead of one's own legs. In empirical research, players of VR games reported significantly higher levels of cybersickness when compared to players on desktop computers (Tan et al., 2015; Yildirim, 2019). Cybersickness is especially severe in games that include running avatars and fast movements in a first-person perspective, such as shooters and role-playing games (Shafer et al., 2019). One solution to potentially reduce the mismatch between virtually perceived and real movement may be newly introduced motion-based VR locomotion systems. 


\section{Locomotion in Virtual Environments}

Locomotion-the players' self-initiated movement within the virtual world (Hale \& Stanney, 2014)-is an essential interaction component in VR with potentially strong effects on elements of the user experience, such as immersion (Bozgeyikli, Raij, Katkoori, \& Dubey, 2016). Further, overcoming the limitations of the physical tracking space to enable a natural, unconstrained movement in VR is regarded as a key component in reducing cybersickness (Nilsson et al., 2018). Even though the latest generation of VR systems works wirelessly, the problem of the limited physical tracking space persists. For example, the maximum playing area for the Oculus Quest is still limited to a maximum size of 25 by 25 feet. Besides these technical limitations, one should also keep in mind that the typical user will most likely not have large enough space available to walk in their home environment in order to explore huge virtual worlds.

One possibility to overcome long distances in virtual worlds is placing the avatar in a moving virtual vehicle (e.g., spaceship or car) steered with hand movements, while the player remains seated-both in the actual and virtual environment. Another option is to put the avatar in a zero-gravity environment to avoid the need for leg movement (e.g., in Mission ISS or Echo Arena). However, both options extensively limit the possibilities of game design. In genres where players typically move around on foot (e.g., shooters, role-playing games, sports simulations), other techniques are needed to solve the locomotion problem.

Boletsis (2017, p. 12) provides a typology of the most commonly used VR locomotion techniques. They can be distinguished by several criteria, for instance the interaction type (physical vs. artificial), the VR motion type (continuous vs. non-continuous) and the VR interaction space (open vs. limited). Based on these criteria, he describes four types of VR locomotion: (1) motion-based locomotion (physical, continuous, open, e.g., walking in place, redirected walking, arm-swinging, zero-gravity); (2) roomscale-based locomotion (physical, continuous, limited, e.g., real walking); (3) controller-based locomotion (artificial, continuous, open, e.g., joystick motion, head-directed); and (4) teleportation-based (artificial, non-continuous, open, e.g., point-and-teleport techniques).

Of these four types of VR locomotion, especially motion-based locomotion is considered a promising approach to solve the above-mentioned problems of VR locomotion in relation to the physical tracking space available to players. In recent years, such motion-based locomotion systems were developed that track players' physical movements and directly transfer them into the virtual world. These systems are believed to cause less cybersickness than artificial, controller-based locomotion (e.g., joystick, head-directed), as the illusory mismatch between perceived and actual movement is seemingly smaller (e.g., Boletsis, 2017; Shafer et al., 2019).
Players perform more extensive movements with bigger body parts (e.g., arms, legs) than in artificial forms of locomotion (e.g., thumbs, head). Continuous motion is more realistically resembling real-world motions that would be performed to achieve a specific movement than, for instance, point-and-teleport techniques.

To enable continuous movement in VR, players have to either walk in place or have to be redirected or repositioned to not reach the limit of the tracking space. This can be achieved through passive repositioning systems that centrally restrain the player in the tracking space by cancelling out forward leg-movement forces with a friction-free platform or an omnidirectional treadmill. Warren and Bowman (2017, p. 163) describe this as a form of "semi-natural VR-locomotion." Repositioned walking is considered to feel more natural than walking in place, as the user is actually stepping forward (Nilsson et al., 2018).

Although passive repositioning systems are a promising technology thought to improve the user experience and to solve issues related to current VR systems, empirical evidence is still scarce. One of the very few studies that has focused exclusively on passive repositioning systems as a form of VR locomotion was conducted by Warren and Bowman (2017). They evaluated user performance in an experiment with an omnidirectional treadmill-the Virtuix Omni. Their findings indicated that playing with the Omni resulted in more negative affective states and a less positive gaming experience than playing with standard game controllers. However, the study followed an exploratory approach and the informative value is limited by the small sample size $(N=10)$.

\section{The Current Study}

Extending this line of research, the purpose of our study is twofold: We aim to investigate how the use of VR technology in general, and the use of an omnidirectional treadmill influence the gaming experience.

As summarized in Section 2, previous research suggests that the use of VR technology can positively influence some aspects of the gaming experience (e.g., Klimmt et al., 2018; Martel et al., 2015; Shelstad et al., 2017; Tan et al., 2015). Based on the existing literature, we assume that VR gaming leads to higher levels of flow, presence, and enjoyment. Additionally, we investigate the measure of awe that has recently been linked to gaming. Awe is defined as emotional responses, mostly positive, like amazement or impressiveness, in reaction to vast stimuli that require accommodation and lead to meaningful, pleasurable experiences (Possler, Klimmt, \& Raney, 2018). Digital games are thought to have a high potential to inspire awe-and therefore increase the pleasure obtained from gameplay-as they often contain potential elicitors of this state, such as vast landscapes, large buildings, massive enemies, and epic soundtracks (Possler, Klimmt, et al., 2018). These elements could be perceived as being especially impressive when presented 
in VR. To investigate the realism of VR locomotion, we further assess the perception of natural mapping connected to various forms of locomotion controls. Natural mapping is understood as "the ability of a system to map its controls to changes in the mediated environment in a natural and predictable manner" (Steuer, 1992, p. 47). Our hypothesis reads as follows:

H1: Using VR technology leads to increases in (a) flow, (b) presence, (c) enjoyment, (d) awe, and (e) natural mapping in comparison to traditional desktop gaming.

However, previous research has also shown that VR gaming can be accompanied by some specific challenging side effects that may influence the gaming experience. For example, it can cause cybersickness (Tan et al., 2015; Yildirim, 2019), and some studies indicated that gaming in VR decreases players' accuracy and control (Martel et al., 2015; Tan et al., 2015). In general, players reported that VR gaming was experienced as more challenging when compared to desktop gaming (Tan et al., 2015). One reason may be the more physically demanding playing situation when using VR devices: Players usually have to stand while playing and perform more extensive body movements for locomotion than in traditional desktop gaming.

Accordingly, we consider cybersickness and physical exertion as two aspects that may make VR gaming more demanding than traditional desktop gaming. Further, we consider the perceived overall challenge and tension as two general components of the user experience that are often investigated in relation to digital games. We hypothesize:

H2: Using VR technology leads to increases in (a) cybersickness, (b) physical exertion, (c) challenge and (d) tension in comparison to traditional desktop gaming.

The second aim of our study is to investigate how the use of an omnidirectional locomotion system influences the gaming experience in VR. As mentioned, research on new forms of locomotion in VR-like repositioned walkingis still scarce. Further, most existing studies focused on technological aspects (e.g., in-game performance, accuracy, wall-hits; Warren \& Bowman, 2017), while there is little research on the user experience (Boletsis, 2017). Nilsson et al. (2018, p. 14) state that "the community has yet to establish how well these systems perform with respect to factors such as perceived naturalness, spatial performance, task performance, and simulator sickness." Presumably, the use of a passive repositioning system could have positive as well as negative effects on the gaming experience. On the one hand, these systems can potentially reduce cybersickness by minimizing the mismatch between perceived virtual and actual movement (e.g., Shafer et al., 2019). On the other hand, extensive movement that is needed to operate this motion-based form of locomotion may cause even more exhaustion than artificial locomotion in VR, which could negatively impair the gaming experience. Additionally, initial empirical results on the use of these systems are rather discouraging, finding that they lead to negative affective states and further reduce players' performance (Warren \& Bowman, 2017). Given the few existing studies and the opposing findings and assumptions, we pose the following open research question:

RQ1: How does the use of a passive repositioning system influence the gaming experience VR technology provides?

\section{Material and Methods}

\subsection{Study Design and Stimulus Material}

Our study follows a one-factorial experimental design. We used the popular role-playing game The Elder Scrolls V: Skyrim as stimulus material (see Figure 1).

The game is available in a VR and an identical nonVR version. We created a hunting task in which players had to shoot various animals using bow and arrow. The task at hand-embedded into a cover story of collecting food for the avatar's family-was running intensively and resembled a typical gaming situation (quest) in this type of game. To assess our hypotheses and research question, three different experimental conditions were implemented:

(1) Traditional desktop gaming ( $n=65$, see Figure 2 ): In this condition, participants used a 24-inch flat screen and controlled their avatar with a standard keyboard and mouse. This resembles a form of artificial, controllerbased locomotion in a non-VR environment.

(2) VR gaming ( $n=68$, see Figure 3 ): For this condition, we used a popular head-mounted VR systemthe HTC VIVE-with its respective handheld controllers as a form of artificial, controller-based locomotion in a VR environment.

(3) Omni gaming ( $n=70$, see Figure 4 ): In this condition, participants also used the HTC VIVE headset and its handheld controllers but were additionally equipped with an omnidirectional treadmill as a passive repositioning system in order to resemble a motionbased, physical form of locomotion in VR. We chose the Virtuix Omni, one of the field's market leaders, for two reasons: First, after its market introduction, this specific omnidirectional treadmill received highly positive reviews from consumer-oriented media (e.g., Carbotte, 2016; Ray, 2016; Tarantola, 2016), calling the Omni "the future of gaming" (Lang, 2013) and proclaiming the addition of a "fantastic new layer of immersion to the virtual reality experience" (Fulton, 2016). Second, to provide comparable results across studies, it is essential to focus on hardware settings that have been studied in previous research (e.g., Warren \& Bowman, 2017). 


\section{COGITATIO}

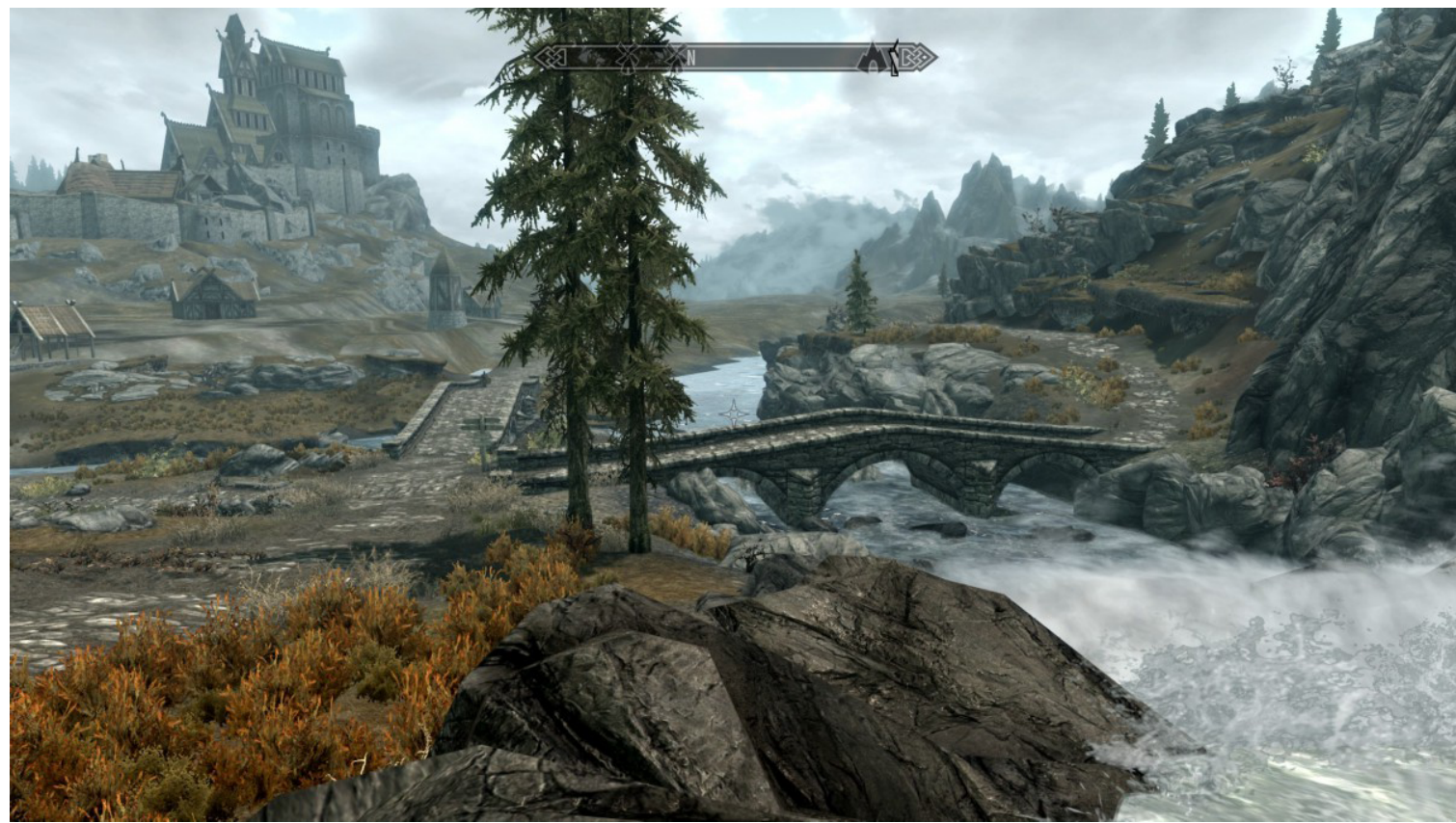

Figure 1. In-game screenshot of The Elder Scrolls V: Skyrim. Source: Livingston (2011).

\subsection{Procedure}

Participants first completed a questionnaire that included measures for randomization checks and sociodemographic variables. They were then randomly assigned to one of the three experimental conditions. Participants were given a three-minute training session to familiarize with the locomotion controls, then they performed the main gaming task in Skyrim for seven minutes. After playing, participants completed a second questionnaire containing the dependent variables, were debriefed, and received a participation compensation of $10 €$.

\subsection{Measures}

We selected established scales to measure the different constructs. Some English original scales were translated to German with the help of a professional translation service.

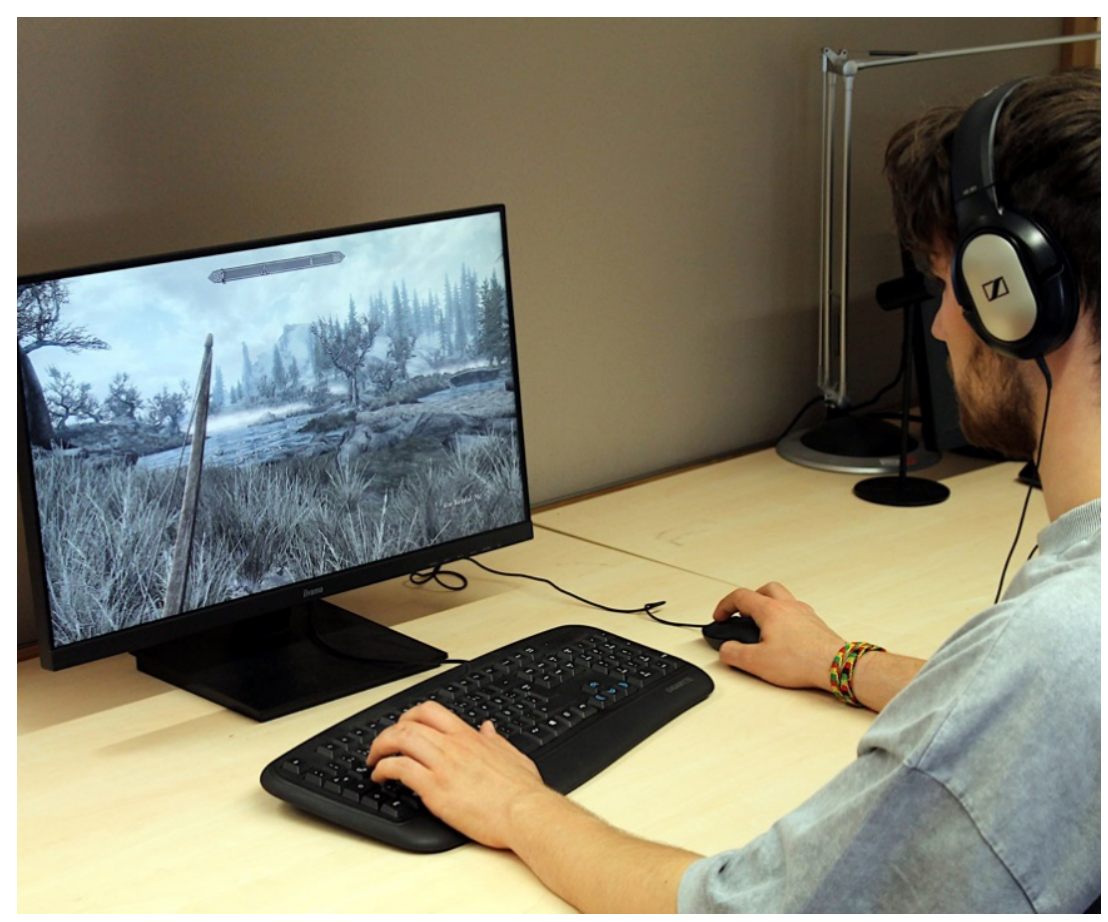

Figure 2. Laboratory setup of the traditional desktop gaming experimental condition. 


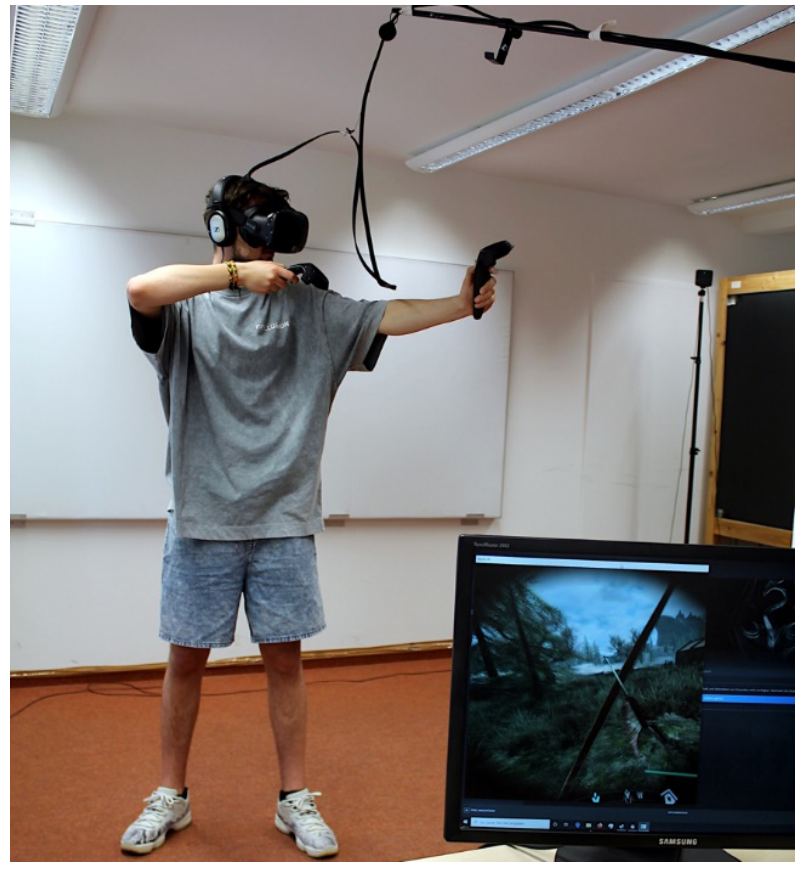

Figure 3. Laboratory setup of the VR gaming experimental condition.

Cybersickness was measured with six items from the ITC-Sense of Presence Inventory by Lessiter, Freeman, Keogh, and Davidoff (2001). Participants rated statements (e.g., "I felt dizzy") on a scale ranging from $1=$ "strongly disagree" to $5=$ "strongly agree." The scale showed satisfactory reliability $(\alpha=.80, M=1.97$, $S D=.82)$

To assess physical exertion, we used the German translation (Löllgen, 2004) of Borg's RPE (Borg, 1970). It consists of a single item ("Please indicate on the scale below how physically exhausted you felt while playing") measured on a scale ranging from $6=$ "no exertion" to $20=$ "maximal exertion" $(M=11.16, S D=3.45)$.

Presence was measured using items of the MEC Spatial Presence Questionnaire by Vorderer et al. (2004). Two different dimensions of presence were considered, both measured with eight items using a scale ranging from $1=$ "strongly disagree" to $5=$ "strongly agree": selflocation (e.g., "I felt like I was actually there in the environment of the presentation") and possible actions (e.g., "It seemed to me that I could have some effect on things in the presentation, as I do in real life"). Both subscales showed high reliability (self-location: $\alpha=.94, M=3.32$, $S D=.99$; possible actions: $\alpha=.86, M=3.20, S D=.81$ ).

To measure flow (e.g., "I forgot everything around me"), challenge (e.g., "I felt challenged"), and tension (e.g., "I felt frustrated"), participants rated their agreement to six items per construct from the German version (Nacke, 2009) of the Game Experience Questionnaire (IJsselsteijn, de Kort, \& Poels, 2008). The items were measured using a scale ranging from $1=$ "strongly disagree" to $5=$ "strongly agree" (flow: $\alpha=.84, M=3.34, S D=.85$; tension: $\alpha=.80, M=2.47, S D=.83$; challenge: $\alpha=.65$, $M=3.04, S D=.71)$.

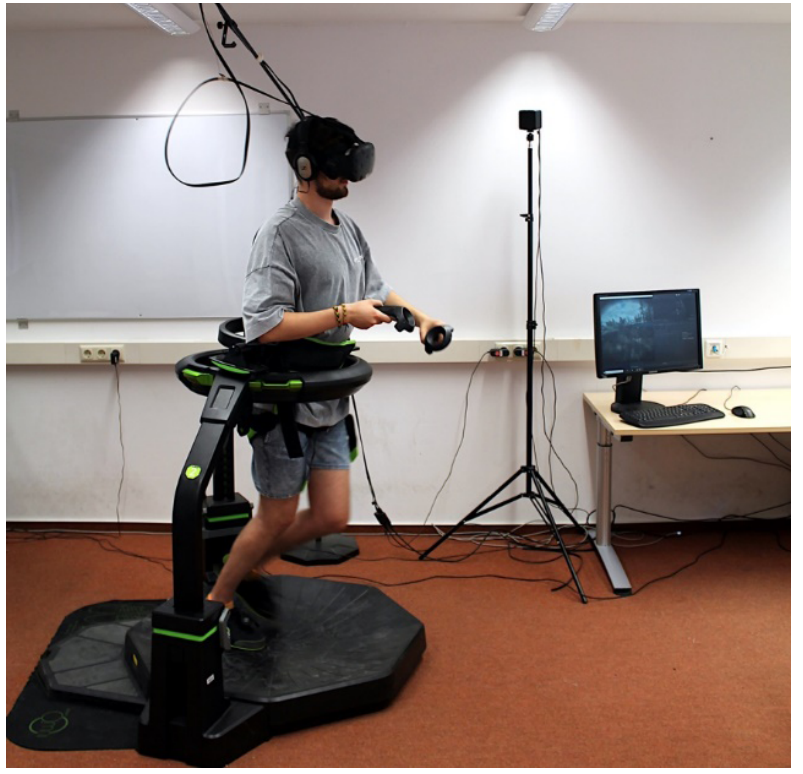

Figure 4. Laboratory setup of the Omni gaming experimental condition.

To measure awe experienced while playing, we used three items originally developed by Possler, Kuempel, Unkel, and Klimmt (2018). Participants rated their agreement to the items (e.g., "I was amazed by the vast and 'epic' gaming experience") on a scale ranging from $1=$ "strongly disagree" to $5=$ "strongly agree." The scale showed satisfactory reliability $(\alpha=.71, M=3.18$, $S D=1.05)$.

We assessed natural mapping with a translated version of the six-item scale by Skalski, Tamborini, Shelton, Buncher, and Lindmark (2011). Participants rated their agreement (e.g., "The game controls seemed natural") on a scale ranging from $1=$ "strongly disagree" to 7 = "strongly agree." The scale showed a high reliability $(\alpha=.90, M=4.17, S D=1.36)$.

To measure enjoyment, we adapted the scale of Ryan, Mims, and Koestner (1983) to the context of gaming. In this six-item scale, participants rated their agreement with statements (e.g., "I enjoyed playing this game very much") on a scale ranging from 1 = "strongly disagree" to 7 = "strongly agree." Two negatively framed items were reverse coded before scale construction. The scale showed a satisfactory reliability $(\alpha=.94, M=5.05$, $S D=1.54)$.

Additional measures were used to check for a successful randomization: Game skill was measured with four items developed by Bracken and Skalski (2006) and translated by Pietschmann (2014). Participants rated items (e.g., "I am a very good computer game player") on a scale ranging from 1 = "strongly disagree" to $5=$ "strongly agree." The scale showed a high reliability ( $\alpha=.90, M=2.69, S D=1.04)$. Participants' physical fitness level was assessed with the five-item International Fitness Scale by Ortega et al. (2011) that proved to be highly reliable ( $\alpha=.83, M=3.64, S D=.68)$. Participants rated statements (e.g., "Your overall physical fitness is...") 
on a scale ranging from $1=$ "very poor" to $5=$ "very good." Participants' prior experience with VR technology was measured with a self-report single item ("I am...in using VR systems") ranging from $1=$ "completely inexperienced" to $5=$ "very experienced" $(M=1.87, S D=.90)$.

Whenever a construct was measured with a scale consisting of several items, we assessed the scale's score by calculating the mean of the scale's items.

\subsection{Sample}

Our sample consisted of 203 participants. They were recruited mainly at a large university in Western Germany through university newsletters and mailing lists, recruitment posters in public places, and a newspaper advertisement. Participants were relatively young $(M=24.18, S D=4.02$, Min. = 18, Max. = 51) and highly educated (93.6\% students; highest educational degree: $39.9 \%$ university degree; $58.6 \%$ high school). Gender was equally distributed ( $49.8 \%$ female). We checked for obvious straight lining in our data set (participants with $>50 \%$ of scales with $S D=0$ ), but no cases had to be excluded as a consequence. As randomization checks, we calculated $\chi^{2}$-tests for group differences in relation to gen$\operatorname{der}\left(\chi^{2}(2)=.07, p=.967\right)$, education $\left(\chi^{2}(8)=7.07\right.$, $p=.529)$, and occupation $\left(\chi^{2}(12)=11.68, p=.472\right)$. To further test our randomization, we calculated a number of independent one-way ANOVAs: There was no difference among groups in relation to participants' mean age $(F(2,200)=1.48, p=.231)$, weekly hours of digital games use $(M=4.67, S D=6.84, F(2,200)=.03, p=.970)$, game skill $(F(2,200)=1.75, p=.176)$, physical fitness level $(F(2,200)=.002, p=.998)$, or prior VR experience $(F(2,200)=.199, p=.819)$.

\section{Results}

A series of independent one-way ANOVAs were calculated with the SPSS 25 software package to compare the gaming experience induced by the different conditions. As displayed in Table 1, we found that the experimental condition affected all independent variables except for tension.

Bonferroni post-hoc tests were calculated to examine the differences between the three conditions in detail. For variables with unequal variances, the Games-Howell post-hoc test was used.

Our first hypothesis expected VR gaming to improve some aspects of the gaming experience when compared to traditional desktop gaming. As can be seen in Table 1, this was true for flow ( $\mathrm{H} 1 \mathrm{a})$, both subdimensions (self-location and possible actions) of spatial presence (H1b), enjoyment (H1c), awe (H1d), and natural mapping $(\mathrm{H} 1 \mathrm{e})$. For these variables, gaming in either the VR gaming or the Omni gaming condition led to significantly higher values for the respective variable when compared to traditional desktop gaming. Therefore, $\mathrm{H} 1$ is fully confirmed.
Our second hypothesis addressed potentially challenging side effects during gameplay. We expected levels of cybersickness (H2a), physical exertion ( $\mathrm{H} 2 \mathrm{~b}$ ), challenge $(\mathrm{H} 2 \mathrm{C})$, and tension $(\mathrm{H} 2 \mathrm{~d})$ to increase in the conditions using VR technology. $\mathrm{H} 2$ was partially confirmed: We found a significant increase in the levels of reported cybersickness ( $\mathrm{H} 2 \mathrm{a}$ ) in both the VR gaming and the Omni gaming condition in comparison to traditional desktop gaming technology (see Table 1). Gaming in VR also led to significantly higher physical exertion $(\mathrm{H} 2 \mathrm{~b})$ than gaming in front of a desktop screen and was perceived as more challenging $(\mathrm{H} 2 \mathrm{C})$. Regarding tension $(\mathrm{H} 2 \mathrm{~d})$, no significant difference between desktop gaming, VR gaming, and Omni gaming was found.

Assessing our exploratory research question on how the use of a passive repositioning system would influence the gaming experience (RQ1), we found that the use of such a system did not differ from other forms of locomotion (see Table 1). We detected no significant differences between VR gaming and Omni gaming in relation to both subdimensions of spatial presence (self-location and possible actions), flow, awe, natural mapping, game enjoyment, cybersickness, challenge, and tension. The only significant difference between VR gaming and Omni gaming was detected in the reported level of received physical exertion. Participants that played in the Omni gaming condition reported significantly higher values of received physical exertion than those in the VR gaming condition.

\section{Discussion and Conclusions}

With regard to both hypotheses, our results support previous research findings (e.g., Klimmt et al., 2018; Martel et al., 2015; Shelstad et al., 2017; Tan et al., 2015) on the influence of VR technology for gaming when compared to more traditional game settings using a desktop computer with flat-screen monitor and mouse and keyboard as input. VR gaming led to higher reported values on several gaming experience measures. Participants reported significantly higher levels of spatial presence (in both subdimensions self-location and possible actions), flow, awe, and enjoyment. They also reported that the controls in VR gaming (i.e., the HTC VIVE controller) felt significantly more natural in comparison to mouse and keyboard. We did not find a difference between desktop and VR gaming in relation to tension. However, in accordance with findings of previous research (e.g., Tan et al., 2015; Yildirim, 2019), gaming in VR led to significantly more cybersickness among participants and was perceived as more challenging.

Passive repositioning systems are believed to reduce cybersickness in VR gaming through fewer sensory conflicts between actual and perceived movements (Boletsis, 2017; Shafer et al., 2019). However, there were no significant differences in reported cybersickness between gaming in VR with and without the Omni. Furthermore, the Omni did not feel more (or less) natural to participants as a locomotion control. Finally, regarding 
Table 1. Detailed results of the independent one-way ANOVAs.

\begin{tabular}{|c|c|c|c|c|c|c|c|c|c|c|c|c|c|c|}
\hline \multirow[t]{3}{*}{ Measure } & \multirow[b]{3}{*}{$d f_{1}$} & \multicolumn{4}{|c|}{ Total model } & \multicolumn{6}{|c|}{ Conditions } & \multicolumn{3}{|c|}{ Differences between conditions ${ }^{a}$} \\
\hline & & \multirow[b]{2}{*}{$d f_{2}$} & \multirow[b]{2}{*}{$F$} & \multirow[b]{2}{*}{$p$} & \multirow[b]{2}{*}{$\omega^{2}$} & \multicolumn{2}{|c|}{$\begin{array}{l}\text { Desktop gaming } \\
\quad(n=65)\end{array}$} & \multicolumn{2}{|c|}{$\begin{array}{l}\text { VR gaming } \\
\qquad(n=68)\end{array}$} & \multicolumn{2}{|c|}{$\begin{array}{l}\text { Omni gaming } \\
\quad(n=70)\end{array}$} & \multirow{2}{*}{$\begin{array}{c}\text { Desktop } \\
\text { vs. VR } \\
\text { gaming }\end{array}$} & \multirow{2}{*}{$\begin{array}{l}\text { Desktop } \\
\text { vs. Omni } \\
\text { gaming }\end{array}$} & \multirow{2}{*}{$\begin{array}{c}\text { VR } \\
\text { vs. Omni } \\
\text { gaming }\end{array}$} \\
\hline & & & & & & $M$ & $S D$ & $M$ & $S D$ & $M$ & $S D$ & & & \\
\hline Cybersickness & 2 & 129.23 & $19.50^{b}$ & $<.001$ & .15 & 1.55 & .56 & 2.21 & .81 & 2.13 & .91 & $* * * d$ & $* * * d$ & n.s. ${ }^{d}$ \\
\hline Received perception of exertion & 2 & 125.74 & $125.64^{\mathrm{b}}$ & $<.001$ & .55 & 8.38 & 2.45 & 10.72 & 3.08 & 14.17 & 1.82 & $* * * d$ & $* * * d$ & $* * * d$ \\
\hline \multicolumn{15}{|l|}{ Spatial presence } \\
\hline Self-location & 2 & 200 & 38.48 & $<.001$ & .27 & 2.56 & .90 & 3.69 & .85 & 3.66 & .80 & $* * * \mathrm{c}$ & $* * * \mathrm{c}$ & n.s. ${ }^{c}$ \\
\hline Possible actions & 2 & 200 & 21.85 & $<.001$ & .17 & 2.70 & .79 & 3.47 & .69 & 3.39 & .73 & $* * * \mathrm{c}$ & $* * * \mathrm{c}$ & n.s. ${ }^{c}$ \\
\hline Flow & 2 & 129.70 & $13.16^{\mathrm{b}}$ & $<.001$ & .11 & 2.93 & .87 & 3.60 & .64 & 3.47 & .87 & $* * * d$ & $* * * d$ & n.s. ${ }^{d}$ \\
\hline Challenge & 2 & 130.09 & $6.98^{b}$ & $<.01$ & .06 & 2.78 & .78 & 3.10 & .69 & 3.23 & .59 & $* d$ & $* * d$ & n.s. ${ }^{d}$ \\
\hline Tension & 2 & 200 & 2.37 & .096 & .01 & 2.40 & .86 & 2.36 & .84 & 2.64 & .79 & n.s. ${ }^{c}$ & n.s. ${ }^{c}$ & n.s. ${ }^{c}$ \\
\hline Awe & 2 & 200 & 49.64 & $<.001$ & .32 & 2.31 & .93 & 3.63 & .80 & 3.56 & .85 & $* * * \mathrm{c}$ & $* * * \mathrm{c}$ & n.s. ${ }^{c}$ \\
\hline Natural mapping & 2 & 200 & $36.18^{b}$ & $<.001$ & .26 & 3.16 & 1.26 & 4.75 & 1.18 & 4.55 & 1.08 & $* * * \mathrm{c}$ & $* * * \mathrm{c}$ & n.s. ${ }^{c}$ \\
\hline Game enjoyment & 2 & 130.12 & $5.09^{b}$ & $<.01$ & .04 & 4.54 & 1.62 & 5.37 & 1.60 & 5.21 & 1.27 & $* d$ & $* d$ & n.s. ${ }^{d}$ \\
\hline
\end{tabular}

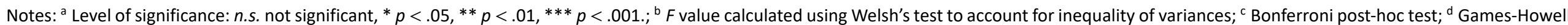
post-hoc test. 
all measures of the gaming experience under study, there were no significant differences between Omni gaming and regular VR gaming. Therefore, we do not find extensive additional benefits of using a passive repositioning omnidirectional treadmill to control locomotion in VR games.

There was only one significant difference between controller-based VR gaming and VR gaming with the Omni: The latter was perceived as being significantly more physically exhausting during gameplay. One might see a utilization potential in the context of exergaming (Oh \& Yang, 2010). As "the ability to tie video games and exercise into a single medium for the benefit of making exercise fun" (Klein \& Simmers, 2009, p. 35), exergaming is believed to be more beneficial for players' physical and cognitive health than sedentary (button-pressing) gaming. Meta-analytical results (Best, 2013) support these hopes placed into the beneficial potential of exergaming. Further, an extensive body of literature describes the beneficial effects of combining sports equipment for physical training (like monodirectional gym-style treadmills) with an immersive gaming environment, usually displayed on standard flat-screen technology. These systems have been used to, for instance, improve stroke patients' balance skills (Yang et al., 2011) or to reduce the risk of falling for elderly people (Mirelman et al., 2016) by projecting the physical workout into a virtual environment that users may perceive as more realistic, natural, and enjoyable than when using standard workout equipment without an immersive component. Further, those systems have proven to support exercise motivation for overweight children who perceived their workout to be more enjoyable, as the immersive gaming component was able to distract their attention away from negative bodily sensations (Baños et al., 2016). Yet, these systems to date rely on monodirectional treadmills and flat-screen technology with a relatively low immersive potential. Our study showed that using VR technology in comparison to screen-based gaming significantly increased presence and enjoyment of our participants. Moreover, adding the Virtuix Omni, an omnidirectional VR treadmill, as locomotion device did not negatively impair participants' gaming experience, even though it was perceived more physically demanding and is less sedentary in nature. Therefore, gaming in VR with the Omni could be used as a form of beneficial physical exercise that is perceived as fun. At the same time, being potentially more immersive than a flat-screen monitor mounted to a standard monodirectional treadmill, we see the potential that the Omni gaming setup could further increase the above-mentioned positive effects of exergaming. We therefore suggest further research on the utilization potential of omnidirectional VR treadmills as an exergaming tool and especially see adolescents and the older gaming population as potential target groups worth to be studied in this context.

In recent times, science enthusiasts, consumer media, and the games industry consider VR technology to be one of the most important innovations in the games sector and express hopes that passive repositioning systems like the Virtuix Omni might help to solve the current problems of VR gaming. Our results only partially support this notion by showing that VR technology indeed improves some aspects of the gaming experience. However, other aspects such as unconstrained, continuous, and natural locomotion in VR as well as cybersickness remain a challenge for game designers. Passive repositioning systems are seemingly not the ideal and final solution for these problems: either because they are not fully developed yet, or because they do not truly allow for natural movement within the virtual world. Therefore, alternative physical locomotion techniques like redirected walking should be further examined. They are thought to allow for natural and continuous movement in an open world in a physical playing area of about 6 by 6 meters by slightly rotating the virtual world around the player (Nilsson et al., 2018; Suma, Azmandian, Grechkin, Phan, \& Bolas, 2015). In combination with novel wireless VR systems (e.g., the Oculus Quest) that offer an expanded playing area in comparison to wire-operated head-mounted displays, this locomotion technique seems fruitful for further exploration. Another direction for future studies could be the combination of VR and treadmill gaming with other innovative peripheral devices, such as gaming vests, to achieve even higher levels of immersion.

Our study has some limitations. The first limitation is the rather homogenous student sample. It is possible that older participants may perceive an increased level of physical exertion as having a greater negative impact on their gaming experience than their younger counterparts. Therefore, it seems promising to replicate our study design with more diverse samples. Also, it cannot completely be ruled out that our results would have been slightly different with another game than Skyrim (VR) as stimulus material. The genre of adventure role-playing games includes a substantial amount of first-person movement while other genres like strategy/simulation or logical thinking games may be less running-intensive. Therefore, we suggest to conduct similar studies with games from various genres in the future. Further, as our respondents were relatively inexperienced in the use of VR technology, the positive perception of and excitement connected to VR technology in relation to gaming as well as the perception of certain aspects of the gaming experience in VR might be partly caused by novelty effects (e.g., Smith \& Du'Mont, 2009). For example, awe might be a concept that could possibly be influenced by such novelty effects. To address this limitation in future research, longitudinal designs or a more experienced sample are recommended.

\section{Acknowledgments}

The authors acknowledge support from the Open Access Publication Fund of the University of Muenster. They 
would like to thank Steve Bernard, Valentin Döring, and Patrick Williams for their help with setting up and conducting some of the field work, all participants of the study for contributing to the research, and the four anonymous reviewers for their thoughtful comments on an earlier version of the manuscript.

\section{Conflict of Interests}

The authors declare no conflict of interests.

\section{References}

Baños, R. M., Escobar, P., Cebolla, A., Guixeres, J., Alvarez Pitti, J., Lisón, J. F., \& Botella, C. (2016). Using virtual reality to distract overweight children from bodily sensations during exercise. Cyberpsychology, Behavior, and Social Networking, 19(2), 115-119.

Best, J. R. (2013). Exergaming in youth: Effects on physical and cognitive health. Zeitschrift für Psychologie, 221(2), 72-78.

Boletsis, C. (2017). The new era of virtual reality locomotion: A systematic literature review of techniques and a proposed typology. Multimodal Technologies and Interaction, 1(24), 1-17.

Borg, G. A. V. (1970). Perceived exertion as an indicator of somatic stress. Scandinavian Journal of Rehabilitation Medicine, 2(2), 92-98.

Bozgeyikli, E., Raij, A., Katkoori, S., \& Dubey, R. (2016). Point \& teleport locomotion technique for virtual reality. In A. Cox, Z. A. Toups, R. L. Mandryk, \& P. Cairns (Eds.), Proceedings of the 2016 annual symposium on computer-human interaction in play (pp. 205-216). New York, NY: Association for Computing Machinery.

Bracken, C. C., \& Skalski, P. (2006). Presence and video games: The impact of image quality and skill level. Paper presented at the 9th Annual International Workshop on Presence, Cleveland, OH, USA.

Carbotte, K. (2016, January 12). Virtuix proved that the Omni VR treadmill is the real deal. tom's HARDWARE. Retrieved from https://www.tomshardware. com/uk/news/virtuix-omni-impresses-th-writers, 30965.html

Csíkszentmihályi, M. (1990). Flow: The psychology of optimal experience. New York, NY: Harper.

Dennison, M. M., Wisti, A. Z., \& D'Zmura, M. (2016). Use of physiological signals to predict cybersickness. Displays, 44, 42-52.

Fulton, W. (2016, January 11). Virtuix Omni hands-on: Walk across virtual worlds from the comfort of your living room. Digital Trends. Retrieved from https://www.digitaltrends.com/gaming/virtuixomni-hands-on

Hale, K. S., \& Stanney, K. M. (2014). Handbook of virtual environments: Design, implementation, and applications. Boca Raton, FL: CRC Press.

IJsselsteijn, W. A., de Kort, Y. A. W., \& Poels, K. (2008).
Game experience questionnaire. Eindhoven: Eindhoven University of Technology.

Klein, M. J., \& Simmers, C. S. (2009). Exergaming: Virtual inspiration, real perspiration. Young Consumers: Insight and Ideas for Responsible Marketers, 10(1), 35-45.

Klimmt, C., Possler, D., \& Steger, F. (2018). True presence, finally: The effect of virtual reality on video game enjoyment. Paper presented at the 68th Annual Conference of the International Communication Association, Prague, Czech Republic.

Lang, B. (2013, April 19). Latest Virtuix Omni VR treadmill video shows intense TF2 gameplay with Oculus Rift and new prototype. Road to VR. Retrieved from https://www.roadtovr.com/virtuix-omniomnidirectional-treadmill-oculus-rft-tf2-gameplay

Lessiter, J., Freeman, J., Keogh, E., \& Davidoff, J. (2001). A cross-media presence questionnaire: The ITCSense of presence inventory. Presence: Teleoperators and Virtual Environments, 10(3), 282-297.

Livingston, J. (2011, November 16). Skyrim [Screenshot from Bethesda Game Studio's The Elder Scrolls V: Skyrim]. Flickr. Retrieved from https://www.flickr. com/photos/xavierwilkinson/6350803784/in/album72157628105061620

Löllgen, H. (2004). Das Anstrengungsempfinden (RPE, Borg-Skala) [Received perception of exertion (RPE, Borg Scale)]. Deutsche Zeitschrift für Sportmedizin, 55(11), 299-300.

Martel, E., Su, F., Gerroir, J., Hassan, A., Girouard, A., \& Muldner, K. (2015). Diving head-first into virtual reality: Evaluating HMD control schemes for VR games. In J. P. Zagal, E. MacCallum-Stewart, \& J. Togelius (Eds.), Proceedings of the 10th international conference on the foundations of digital games (pp. 1-5). Santa Cruz, CA: Society for the Advancements of the Science of Digital Games.

McCauley, M. E., \& Sharkey, T. J. (1992). Cybersickness: Perception of self-motion in virtual environments. Presence: Teleoperators and Virtual Environments, 1(3), 311-318.

Mirelman, A., Rochester, L., Maidan, I., Del Din, S., Alcock, L., Nieuwhof, F., . . . Hausdorff, J. M. (2016). Addition of a non-immersive virtual reality component to treadmill training to reduce fall risk in older adults (V-TIME): A randomised controlled trial. The Lancet, 388(10050), 1170-1182.

Nacke, L. (2009). Affective ludology: Scientific measurement of user experience in interactive entertainment (Unpublished doctoral dissertation). Blekinge Institute of Technology, Karlskrona, Sweden.

Nilsson, N. C., Serafin, S., Steinicke, F., \& Nordahl, R. (2018). Natural walking in virtual reality: A review. ACM Computers in Entertainment, 16(2), 1-22.

Oh, Y., \& Yang, S. P. (2010). Defining exergames \& exergaming. In MSU Serious Games Program (Ed.), Proceedings of the meaningful play 2010 conference (pp. 1-18). East Lansing, MI: Meaningful Play. 
Ortega, F. B., Ruiz, J. R., España-Romero, V., VicenteRodriguez, G., Martinez-Gómez, D., Manios, Y., . . . Castillo, M. J. (2011). The International Fitness Scale (IFIS): Usefulness of self-reported fitness in youth. International Journal of Epidemiology, 40(3), 701-711.

Pietschmann, D. (2014). Spatial Mapping in virtuellen Umgebungen: Der Einfluss von Stereoskopie und Natural Mapping auf die User Experience [Spatial mapping in virtual environments: The influence of stereoscopy and natural mapping on user experience] (Unpublished doctoral dissertation). University of Technology Chemnitz, Chemnitz, Germany.

Possler, D., Klimmt, C., \& Raney, A. A. (2018). Gaming is awesome! A theoretical model on cognitive demands and the elicitation of awe during video game play. In N. D. Bowman (Ed.), Video games: A medium that demands our attention (pp. 74-91). London: Routledge.

Possler, D., Kuempel, A. S., Unkel, J., \& Klimmt, C. (2018). Awe-inspiring video games: Exploring the role of the emotion awe for video game entertainment. Paper presented at the 7th Conference of the European Communication Research and Education Association, Lugano, Switzerland.

Ray, T. (2016, January 8). Virtuix's VR treadmill is a bit like a drug trip, and a good one. Barron's. Retrieved from https://www.barrons.com/articles/virtuixs-vrtreadmill-is-a-bit-like-a-drug-trip-and-a-good-one1452286957

Ryan, R. M., Mims, V., \& Koestner, R. (1983). Relation of reward contingency and interpersonal context to intrinsic motivation: A review and test using cognitive evaluation theory. Journal of Personality and Social Psychology, 45(4), 736-750.

Sanders, T., \& Cairns, P. (2010). Time perception, immersion and music in videogames. In T. McEwan \& L. M. McKinnon (Eds.), Proceedings of the 24th BCS interaction specialist group conference (pp. 160-167). Swindon: BCS.

Shafer, D. M., Carbonara, C. P., \& Korpi, M. F. (2019). Factors affecting enjoyment of virtual reality games: $\mathrm{A}$ comparison involving consumer-grade virtual reality technology. Games for Health Journal, 8(1), 15-23.

Shelstad, W. J., Smith, D. C., \& Chaparro, B. S. (2017). Gaming on the Rift: How virtual reality affects game user satisfaction. Proceedings of the Human Factors and Ergonomics Society Annual Meeting, 61(1), 2072-2076.

Skalski, P., Tamborini, R., Shelton, A., Buncher, M., \& Lindmark, P. (2011). Mapping the road to fun: Natural video game controllers, presence, and game enjoyment. New Media \& Society, 13(2), 224-242.

Smith, S. P., \& Du'Mont, S. (2009). Measuring the effect of gaming experience on virtual environment navigation tasks. Paper presented at the 2009 IEEE Symposium on 3D User Interfaces, Lafayette, LA, USA.
Steuer, J. (1992). Defining virtual reality: Dimensions determining telepresence. Journal of Communication, 42(4), 73-93.

Suma, E. A., Azmandian, M., Grechkin, T., Phan, T., \& Bolas, M. (2015). Making small spaces feel large: Infinite walking in virtual reality. In Association for Computing Machinery (Ed.), Proceedings of SIGGRAPH 2015 emerging technologies (p. 1). New York, NY: Association for Computing Machinery.

Superdata. (2019). Oculus expected to sell $1.3 \mathrm{M}$ Quest units in 2019; XR revenue reached $\$ 6.6 B$ in 2018 and is projected to increase $442 \%$ by 2022 . Superdata. Retrieved from https://www.superdata research.com/xrupdate

Sweetser, P., \& Wyeth, P. (2005). GameFlow: A model for evaluating player enjoyment in games. ACM Computers in Entertainment, 3(3), 3.

Tan, C. T., Leong, T. W., Shen, S., Dubravs, C., \& Si, C. (2015). Exploring gameplay experiences on the Oculus Rift. In A. L. Cox, P. Cairns, R. Bernhaupt, \& L. Nacke (Eds.), Proceedings of the 2015 annual symposium on computer-human interaction in play (pp. 253-263). New York, NY: Association for Computing Machinery.

Tarantola, A. (2016, June 1). I quite literally ran around a virtual arena and loved it. Engadget. Retrieved from https://www.engadget.com/2016-01-06-i-quiteliterally-ran-around-a-virtual-arena-and-lovedit.html

Viar360. (2019). Virtual reality market size in 2018 with forecast for 2019. Viar360. Retrieved from https://www.viar360.com/virtual-reality-marketsize-2018/

Vorderer, P., Wirth, W., Gouveia, F. R., Biocca, F., Saari, T., Jäncke, F., . . . Jäncke, P. (2004). MEC Spatial Presence Questionnaire (MEC-SPQ): Short documentation and instructions for application. Hannover: Measurement, Effects, Conditions (MEC).

Warren, L. E., \& Bowman, D. A. (2017). User experience with semi-natural locomotion techniques in virtual reality: The case of the Virtuix Omni. In A. L. Simeone, K. Johnsen, R. Teather, \& C. Sandor (Eds.), Proceedings of the 5th symposium on spatial user interaction (p. 163). New York, NY: Association for Computing Machinery.

Witmer, B. G., \& Singer, M. J. (1998). Measuring presence in virtual environments: A presence questionnaire. Presence, $7(3), 225-240$.

Yang, S., Hwang, W.-H., Tsai, Y.-C., Liu, F.-K., Hsieh, L.F., \& Chern, J.-S. (2011). Improving balance skills in patients who had stroke through virtual reality treadmill training. American Journal of Physical Medicine and Rehabilitation, 90(12), 969-978.

Yildirim, C. (2019). Cybersickness during VR gaming undermines game enjoyment: A mediation model. Displays, 59, 35-43. 


\section{About the Authors}
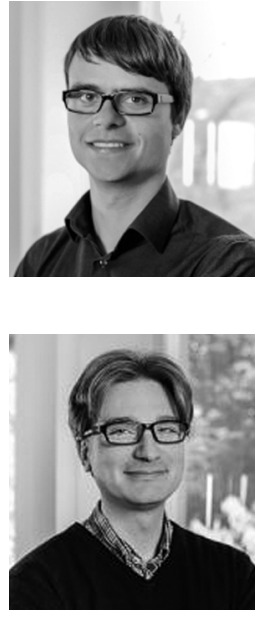

Felix Reer is a Postdoctoral Researcher in the Department of Communication at the University of Muenster, Germany. His research interests include social media and online communication, effects of digital games, and the use of highly immersive media technologies, such as virtual reality and augmented reality devices. He is Vice-Chair of the Digital Games Research Section of the European Communication Research and Education Association (ECREA).
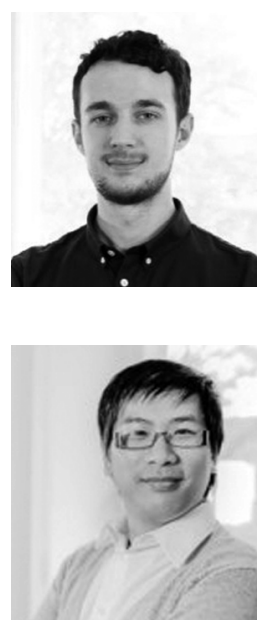

Wai Yen Tang worked as a Postdoctoral Researcher in the Department of Communication at the University of Muenster, Germany, until 2019. He is a Communication Scientist with a background in Psychology. In 2016, he received his PhD in Communication Studies from the Ohio State University in Columbus, OH, USA. His research interests include digital games' influence on behavior and players' online communication, such as sexual harassment.

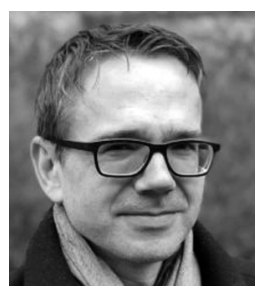

Thorsten Quandt is a Professor of communication studies at the University of Muenster, Germany. His research interests include online communication, digital games, VR, and journalism. 